\title{
SEROPREVALENCIA DE Brucella abortus Y OCURRENCIA DE Brucella melitensis EN CAPRINOS Y EN OVINOS DE CESAR Y SUCRE
}

\author{
SEROPREVALENCE OF Brucella abortus \\ AND OCURRENCE OF Brucella melitensis \\ IN GOATS AND SHEEP OF CESAR AND SUCRE
}

\author{
Vaneza Tique1, Eliana Daza², Jaime Álvarez³, Salim Mattar
}

\begin{abstract}
${ }^{1}$ Bacterióloga. Universidad de Córdoba, Facultad Medicina Veterinaria y Zootecnia, Instituto de Investigaciones Biológicas del Trópico. vtiquesalleg@yahoo.com ${ }^{2}$ Microbióloga. Universidad Popular del Cesar, Facultad de Ciencias de la Salud. Programa Microbiología. emdaza@hotmail.com ${ }^{3}$ MVZ, M.Sc. Universidad de Córdoba, Facultad Medicina Veterinaria y Zootecnia. Instituto de Investigaciones Biológicas del Trópico. jalvarezpt@yahoo.com ${ }^{4}$ Ph.D. Universidad de Córdoba, Facultad Medicina Veterinaria y Zootecnia, Instituto de Investigaciones Biológicas del Trópico. Sede Berástegui Tel/fax: (094)7560710; Montería, Córdoba. http://www.unicordoba.edu. co/institutos/iibt/ Email. mattarsalim@hotmail.com
\end{abstract}

Rev. U.D.C.A Act. \& Div. Cient. 13 (2) 133-139, 2010

\section{RESUMEN}

Brucella melitensis es el principal agente causal de la brucelosis en caprinos y en ovinos. En Colombia, no se han notificado aislamientos o seroreactantes de este agente, razón por la cual, se requiere hacer una vigilancia activa de casos, en poblaciones susceptibles a padecer la enfermedad, por esta u otra especie. El objetivo de este estudio fue el de estimar la seroprevalencia de brucelosis en caprinos y en ovinos, en los municipios de Valledupar (Cesar) y Coloso (Sucre). Se realizó un estudio descriptivo prospectivo de corte transversal, que incluyó un total de 329 animales, entre caprinos y ovinos. En el municipio de Valledupar, se muestrearon 209 caprinos, procedentes de diez predios y, del municipio de Coloso, 120 ovinos originarios de cuatro predios. El diagnóstico serológico, se llevó a cabo por la técnica de aglutinación rápida en placa Rosa de Bengala y la técnica de ELISA indirecta, para la detección de anticuerpos en caprinos y en ovinos. El presente estudio permitió estimar una seroprevalencia a $B$. abortus del $1,2 \%$ (4/329) por la técnica de Rosa de Bengala; estos sueros fueron evaluados por ELISA competitiva, resultando negativos a esta prueba. La seroprevalencia de $B$. melitensis fue del $0 \%$, lo que permitió establecer un riesgo limitado y mínimo, que concuerda con la notificación nunca antes realizada en Colombia. Estos hallazgos confirman la importancia de una vigilancia activa y permanente en estas especies susceptibles.

Palabras clave: Brucelosis, pequeños rumiantes, Rosa de Bengala, Colombia.

\section{SUMMARY}

Brucella melitensis is the main causative agent of brucellosis in goats and sheep. In Colombia, seropositives against $B$. mellitenses has not been detected, therefore, it is important to carry out an active surveillance in these two animal groups that could be susceptible to $B$. mellitenses or other Brucella species. The aim of this study was to determine the seroprevalence of $B$. melitensis and of $B$. abortus in goats in the municipalities of Valledupar (Cesar) and sheep in the municipality of Coloso (Sucre). A prospective study of crosssection that included a total of 329 animals, goats and sheep, was performed. In the municipality of Valledupar 209 goats were sampled, belonging to ten farms and the municipality of Coloso 120 sheep within the four farms. Serologic diagnosis was performed by the technique of rapid agglutination in plate Rose Bengal and indirect ELISA for goats and sheep. This study allowed to estimate seroprevalence to $B$. abortus of $1.2 \%$ (4/329) by the technique of Rose Bengal, these sera were confirmed by competitive ELISA, detecting negative results. The seroprevalence of $B$. melitensis was $0 \%$, thereby allowing to extrapolate a limited risk and agrees with the notification never made in Colombia. These findings confirm the importance of active surveillance in these two susceptible species.

Key words. Brucellosis, small rumiants, Rose Bengal, Colombia. 


\section{INTRODUCCIÓN}

La brucelosis es una de las más importantes y extendidas zoonosis en el mundo, por sus serias implicaciones en salud pública, especialmente, en el aumento de incidentes en humanos, que ascienden a un millón de nuevos casos anuales y tasa de prevalencia, en algunos países, que exceden diez casos por 100.000 habitantes (Franco et al. 2007) y por que también actúa como una barrera sanitaria, para el comercio internacional de animales y de sus productos; el ganado caprino, ovino, bovino y porcino puede ser infectado por Brucella spp. El control y la prevención de la brucelosis en animales es esencial para la erradicación de la enfermedad en el hombre (Seleem et al. 2010; Neta et al. 2010).

Brucella melitensis (biovar 1, 2 y 3 ) es el principal agente causal de la brucelosis caprina. Esta especie es endémica en la región del mediterráneo y, según la Organización Mundial de Sanidad Animal (OIE), la brucelosis caprina por B. melitensis está presente en otros países de América, como México, Argentina, Paraguay, Venezuela y Bolivia, alcanzando en algunas regiones, como México, a superar el 10\%. Estados Unidos, el norte y centro de Europa, sureste de Asia, Australia y nueva Zelandia están libres de este agente (OIE, 2004). La ocurrencia de infecciones naturales por $B$. abortus en caprinos y en ovinos es esporádica.

Patológica y epidemiológicamente, la infección por $B$. melitensis en cabras y en ovejas es muy similar a la infección por $B$. abortus en ganado; sin embargo, esta especie es más invasiva, patógena y fácilmente trasmisible a los humanos. Los síntomas más comunes incluyen fiebre aguda intensa, progresando, a una forma crónica, que afecta la funcionalidad de los músculos esqueléticos, sistema cardiovascular y sistema nervioso central (OIE, 2004).

En Colombia, el Instituto Colombiano Agropecuario ICA, no ha reportado casos por $B$. melitensis; no obstante, existe contagio de caprinos por $B$. abortus, que afecta a los bovinos, principalmente, por lo que los casos de brucelosis en humanos son frecuentes en nuestro país y la brucelosis bovina alcanza una prevalencia del $4 \%$ (Orjuela et al. 2009; Lucero et al. 2008).

En el departamento del Cesar, la explotación del ganado caprino ha sido tradicional y las políticas del gobierno nacional han motivado su aumento en el pie de cría; A pesar de de ello, los departamentos del Cesar y de Sucre no cuentan con un paquete tecnológico para la explotación de estas especies, por lo que su competitividad pecuaria es baja y, por lo tanto, las condiciones sanitarias, son mínimas. La explotación no tecnificada sin control de los rebaños conduce a un descenso en el sector productivo agropecuario y se constituye en un riesgo de salud pública para contraer gérmenes zoonóticos, como $B$. melitensis y $B$. abortus. En los municipios de Valledupar y de Colosó no existen estudios que determinen la prevalencia de $B$. melitensis y $B$. abortus en la población caprina y ovina.

Colombia es considerado un país libre de $B$. melitensis, ya que no existen reportes de circulación de esta especie en el ganado caprino y ovino, evento ratificado en el informe de sanidad del 2006, del Instituto Colombiano Agropecuario (ICA), que no reporta animales positivos a Brucella abortus en caprinos $(n=577)$ y ovinos $(n=481)$, de diferentes predios del territorio nacional (Orjuela et al. 2009). Durante el período 1968-1991, sólo se reportó el aislamiento de $B$. suis $(\mathrm{n}=12)$ y $B$. abortus $(n=79)$ en muestras procedentes de humanos, de bovinos, de caprinos y de ovinos (Lucero et al. 2008).

La especie caprina registra 577.298 animales en el territorio nacional, donde el $45 \%$ está concentrado en el departamento de La Guajira. En Sucre existen 2.986 animales y en el Cesar 36.120. Con respecto a la explotación de la especie ovina en el país, se registran 1.297.118 ejemplares distribuidos, prioritariamente, en los departamentos de La Guajira, Boyacá y Magdalena, que agrupan el $51 \%$. En el departamento de Cesar, la población es de 78.493 y, en Sucre, 29.170. Esta situación reviste de importancia sanitaria, ya que la especie ovina es susceptible a enfermedades epidemiológicamente importantes y cumple un papel decisivo en la supervivencia y en la diseminación de agentes virales, parasitarios o bacterianos (Instituto Colombiano Agropecuario, 2008a).

Las condiciones de alta transmisión y contagio al hombre por parte de Brucella spp., así como su efecto económico y social en el sector agropecuario, hace necesario realizar una vigilancia sobre $B$. abortus y $B$. melitensis en la población creciente de caprinos y de ovinos, en los municipio de Valledupar y de Colosó.El objetivo de este estudio fue estimar la seroprevalencia de brucelosis en estas dos especies, en los municipios de Valledupar (Cesar) y Colosó (Sucre).

\section{MATERIALES Y MÉTODOS}

Se realizó un estudio descriptivo prospectivo de corte transversal, que incluyó un total de 329 caprinos y ovinos, del municipio de Valledupar (caprinos $=209$ ) y del municipio de Colosó (ovinos $=120$ ).

El departamento de Cesar, se encuentra ubicado en el nororiente del país, localizado entre los $07^{\circ} 41^{\prime} 16^{\prime \prime}$ y $10^{\circ} 52$ ' 14 " de latitud norte y los 72'53'27" y 7408'28" de longitud oeste. Hace parte del Caribe Colombiano y su capital es Valledupar, municipio que posee 18 corregimientos, donde once se sitúan en el punto norte y siete, en el área sur. El departamento 
de Sucre también está localizado al norte del país, en la región Caribe Colombiana; localizado entre los $10^{\circ} 08^{\prime} 03^{\prime \prime}$ y

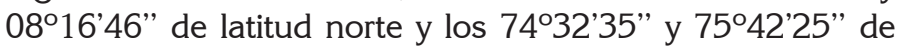
longitud oeste. Su capital es Sincelejo y está conformado por cinco subregiones; en la subregión de los Montes de María fue muestreada la población ovina (Instituto Geográfico Agustín Codazzi, 2010).

Según datos relacionados en el Censo (ICA 2008a), la población de ovinos $(n=29.170)$ y de caprinos $(n=36.120)$, existentes en los departamentos de Sucre y de Cesar, fueron tenidos en cuenta para calcular el tamaño de la muestra y se consideró una frecuencia esperada del $10 \%$, según estudios previos (Solorio et al. 2007; Taboada et al. 2005), un error máximo permisible del $5 \%$ y un nivel de significancia del $5 \%$. Con base en estos datos, el tamaño de la muestra fue de 138 para cada una de las especies; sin embargo, se tomaron 209 muestras de caprinos y 120 de ovinos.

En el municipio de Valledupar, se incluyeron diez de los principales predios productores de ganado caprino de los 120 existentes en el norte y en el sur del municipio, dedicados a la labor de explotación caprina no tecnificada; la mayoría, propiedad de pequeños productores. Se incluyó, el $61 \%(n=7)$ de los corregimientos del norte y $39 \%(n=3)$ de los del sur; cada predio tenía 24 hembras de tipo reproductor y un macho.

En el municipio de Colosó (Sucre), se muestrearon cuatro de las principales explotaciones ovinas, ubicadas en zonas rurales denominadas el Paraíso, Umata, el Rodeo y las Delicias, donde se muestrearon un total de 120 animales.

La toma de muestras de sangre total fue realizada de la vena yugular o en la coccígea, conservadas en refrigeración y después congeladas a $-20^{\circ} \mathrm{C}$, para su posterior análisis serológico, en el Instituto de Investigaciones Biológicas del Trópico de la Universidad de Córdoba. El diagnóstico serológico para Brucelosis, se llevó a cabo por la técnica de aglutinación rápida en placa Rosa de Bengala, con un antígeno coloreado, acidificado y tamponado. Es una suspensión concentrada de B. abortus (Cepa S99 Weybridge), concentración celular 7,5\% - 8,5\%, inactivada por el calor y el fenol al 0,5\%, diluida en tampón ácido $(\mathrm{pH} 3,6)$ y coloreada con Rosa de Bengala (Instituto Pourquier, Montpellier, Francia). El antígeno esta calibrado para dar reacción positiva a una dilución 1:45 y reacción negativa a la dilución 1:55, utilizando el suero estándar internacional, de la oficina internacional de epizootias. Se realizó la respectiva modificación a los volúmenes de reacción para la especie caprina y ovina $(75 \mu \mathrm{L}$ de suero y $25 \mu \mathrm{L}$ de antígeno), recomendada para mejorar la sensibilidad de la prueba (OIE, 2004). Los animales que resultaron positivos a esta prueba fueron confirmados por la técnica de ELISA Competitiva (Svanova, Svanovir ${ }^{\mathrm{TM}}$, Uppsala,
Sweden), realizadas en el Centro Integral de Sanidad Animal (CISA), del departamento de Córdoba.

Para la detección de anticuerpos de $B$. melitensis (IgG1), se utilizó la técnica de ELISA indirecta estandarizada para el diagnóstico en la especie caprina y ovina (Brucelisa 160SG +400SG Veterinary Laboratories Agency, UK).

Los procedimientos de toma de muestra, de manejo y de conservación, se realizaron teniendo en cuenta las normas éticas, técnicas, científicas y administrativas para la investigación en animales, según la ley 84 (Congreso Nacional de Colombia, 1989). Durante el estudio, se mantuvo la confidencialidad de la información de predios muestreados y de animales positivos. El estudio fue aprobado previamente por el comité de ética del Instituto de Investigaciones Biológicas del Trópico de la Universidad de Córdoba, mediante la resolución 012008 (Enero de 2008).

Los datos recolectados en la aplicación de una encuesta a propietarios o encargados de los predios fueron tabulados en una hoja electrónica de MS Excel®. Se realizó un análisis descriptivo de las variables y, finalmente, se cruzaron las diversas variables (edad, predio, vacunación), para establecer la relación con la presencia de $B$. abortus y $B$. melitensis. El análisis estadístico fue realizado por medio de los programas SPSS 10 y Statgraphics.

\section{RESULTADOS Y DISCUSIÓN}

La brucelosis en la población caprina y ovina es considerada una enfermedad de menor importancia en algunos países de Latinoamérica; en Colombia, Brucella melitensis no genera una preocupación, ya que no ha sido aislada ni existe evidencia serológica de animales seropositivo a estas especies. En contraste, $B$. abortus biovar 1 es la especie más importante asociada a brucelosis y es considerada un problema de salud pública humana y animal (Lucero et al. 2008).

En el presente estudio, de los sueros de ovinos, sólo el $3,33 \%$ (4/120), procedentes de Sucre, resultaron positivo a la prueba de Rosa de Bengala, pero al ser analizadas con ELISA competitivo fueron negativas, lo cual, sugiere que se trató de reacciones inespecíficas. Todas las muestras de caprinos $(n=326)$ resultaron negativo a las pruebas de Rosa de Bengala y el total de las muestras de ovinos y de caprinos procesadas con la técnica de ELISA indirecto resultaron negativo (Tabla 1).

Los resultados obtenidos permiten establecer un riesgo limitado y mínimo, que concuerda con la notificación nunca antes realizada en Colombia, en contraste con la de otros países suramericanos, como Perú y Argentina (Samartino, 2002, Taboada et al. 2005). En Colombia, no se dispone de 
Tabla 1. Información epidemiológica de la población caprina y ovina estudiada.

\begin{tabular}{|c|c|c|c|c|c|c|c|}
\hline \multirow[t]{3}{*}{ Departamento } & \multicolumn{3}{|c|}{ Seroprevalencia } & \multicolumn{2}{|c|}{ Edad (meses) } & \multirow[t]{3}{*}{ Predios } & \multirow[t]{3}{*}{ Condición } \\
\hline & \multicolumn{2}{|c|}{ B. abortus } & \multirow{2}{*}{$\begin{array}{c}\text { B. melitensis } \\
\text { ELISA Indirecta }\end{array}$} & \multirow[b]{2}{*}{3 a 6} & \multirow[b]{2}{*}{$>6$} & & \\
\hline & $\mathrm{RB}$ & ELISA-C & & & & & \\
\hline Sucre & $4(3,33 \%)$ & 0 & 0 & 3 & 117 & 4 & No estabulado \\
\hline Cesar & 0 & 0 & 0 & 0 & 209 & 10 & Semi estabulado \\
\hline Total & $1,2 \%$ & $0 \%$ & $0 \%$ & 3 & 326 & 14 & \\
\hline
\end{tabular}

encuesta nacionales de seroprevalencia de $B$. melitensis, por lo que la estimada fue la esperada; aunque estos hallazgos serológicos requieren ser corroborados con estudios que incluyan el aislamiento bacteriano.

Garro et al. (2005), en estudio adelantado en 392 hembras, pertenecientes a cuatro distritos de la provincia de Barranca, Lima Perú, empleando la prueba de Rosa de Bengala, no encontró animales seropositivos a Brucella spp., situación igual a la hallada en presente estudio.

Los resultados obtenidos de seroprevalencia a $B$. abortus o $B$. melitensis en cabras y en ovejas, en esta investigación, demuestra la ausencia de reactores a Brucella spp. y la posible influencia del sistema de crianza extensiva y sedentaria implementado, que contribuye a un mejor control de los hatos.

Es posible que la presencia de anticuerpos a $B$. abortus en ovinos corresponda a una contaminación cruzada entre especies animales por prácticas pecuarias, donde existe un continuo contacto entre las especies en los predios, ya que han sido reportados aislamientos de B. suis, B. melitensis y $B$. abortus en cabras (Lucero et al. 2008). Se han comprobado que las infecciones en bovinos por $B$. melitensis y $B$. suis interfieren con el diagnóstico serológico de las infección por B. abortus (Neta et al. 2010).

En los países de centro y sur América, la incidencia anual de casos de brucelosis en humanos es de 0,6 a 34,9 casos (por un millón de habitantes), situación por la que es considerada una área endémica de la enfermedad en humanos, con excepción de Perú y de Argentina, donde los hallazgos han disminuido, como resultados de las medidas de control en especies animales susceptibles, como bovinos, cabras y ovejas. En el Brasil, que posee una alta población de caprinos y de ovinos, $B$. melitensis no está presente. Algunos carneros presentan epididimitis causada por $B$. ovis, particularmente, en los estados del sur, en donde la producción ovina es más desarrollada. En estudios recientes, se ha demostrado una ascendente tendencia en la prevalencia de la enfermedad (Poester et al. 2002; Pappas et al. 2006).

En Venezuela, B. melitensis ha sido aislada, pero el impacto en animales y en humanos no es bien conocido, situación que ocurre en muchos de los países, donde $B$. abortus es el principal agente causante de la brucelosis (Vargas, 2002).

Baumgarten (2002), en Paraguay, reportó la presencia de Brucella melitensis biovar 1 y 2 y notificó casos de esta zoonosis con un aislado identificado como $B$. melitensis biovar 1 en un paciente humano adulto y que, probablemente, se infectó por el consumo de leche de cabra. En algunos países de Centro América, se ha constatado la presencia de B. melitensis (Moreno, 2002). En Guatemala, ha sido aislada en ovinos y en humanos y, en Panamá, se han presentado casos sospechosos. En Costa Rica y en El Salvador, el 80\% de la población ovina y caprina no tiene anticuerpos contra Brucella spp., lo que sugiere la ausencia de $B$. melitensis en estos dos países, similar alos hallazgos del presente estudio.

En Argentina, el registro de caprinos representa el $4 \%$ de la población general, que se encuentra concentrada en el noreste del país, región en la cual, recientemente, fue estimada una prevalencia que alcanza el $25 \%$, en la especie caprina, donde ha sido aislada B. melitensis biovar 1 (Samartino, 2002).

Entre los factores de riesgo asociados a la aparición de animales seropositivos a Brucella spp. esta la crianza conjunta con otras especies animales. Esta práctica, además de generar hacinamiento y aumentar el riesgo de infección caprina por inhalación del polvo contaminado de los establos, obliga a realizar la vacunación en otras especies (ganado ovino). Esto requiere de mayor intervención educativa en el personal responsable de los animales, situación que en los hatos muestreados se identificó y, es posible, que facilitara 
la transmisión de animales con infección natural a animales susceptibles, como cabras y ovejas que, probablemente, no desarrollan la enfermedad (Aguiar et al. 2007; Taboada et al. 2005).

Para el diagnóstico oficial de $B$. abortus en las especies bubalina, caprina, canina, equina, ovina y porcina, el ICA dispone de las pruebas de Rosa de bengala, fijación de complemento y ELISA competitiva. La implementación de técnicas especializadas, como la ELISA indirecta, ha permitido confirmar, por primera vez, en el país, la seroprevalencia a $B$. melitensis (0\%), en una población de ovinos y de caprinos, de los departamento de Cesar y de Sucre, estableciendo así una pauta para futuros estudios en estas u otras regiones, ya que la ELISA indirecta existente es exclusiva para el diagnóstico en bovinos (ICA, 2008b). Los animales incluidos en el presente estudio no estaban vacunados contra brucelosis bovina ( $B$. abortus C 19 o RB51) o brucelosis caprina (en Colombia, no está indicado vacunar con $B$. melitensis Rev 1); sin embargo, se observaron cuatro animales positivos a Rosa de Bengala, pero negativos a ELISA competitiva, lo cual, sugiere que otras bacterias generan reacción cruzada, dando falsos positivos.

En otros países, las intervenciones por parte del Estado para controlar la brucelosis caprina son la vacunación a cabras menores de tres meses, con la cepa B. melitensis Rev-1, medida que en Colombia no se ha establecido ni estaría indicada, porque el agente causal no ha sido identificado. Por lo tanto, adquiere relevancia la implementación de un sistema de vigilancia epidemiológica activa, teniendo en cuenta que se ha detectado la enfermedad en países limítrofes y del resto de América del Sur y Central. (Poester, 2002; Samartino, 2002).

El trabajo educativo con los ganaderos se convierte en una medida preventiva de esta enfermedad, que garantizará la implementación de acciones que reducen los riesgos para el personal y otras especies susceptible, presente en los predios afectados. El porcentaje registrado de criadores analfabetas fue del 5\%, en comparación con Taboada et al. (2005), en Perú, quienes en su estudio establecieron un $(11,1 \%)$, cifras no esperadas por ser esta una población rural.

Solorio et al. (2007) realizaron un estudio que incluyó 5.114 cabras, de 79 predios en Michoacan, México, que permitió estimar una seroprevalencia del 9,8\% para $B$. melitensis, cifras superiores a las de la presente investigación. Simultáneamente, evaluaron los factores de riesgo asociados con seropositividad en cabras, como son los animales de rebaños grandes (> 34 animales), rebaños con alta densidad ( $>3,5$ animales $/ \mathrm{m}^{2}$ ) y animales con edad $>24$ meses. Estas variables no fueron evaluadas en el presente trabajo, pero la población estudiada fueron animales jóvenes que, un su mayoría, provenían de rebaños pequeños, donde la densidad de animales es baja. La seroprevalencia estimada de $B$. melitensis $(0 \%)$ no indica que no ha habido exposición al microorganismo, ya que puede ser difícil la detección de la infección por serología en rebaños, con infección prolongada. Se ha descrito que los animales con una larga infección siguen siendo serológicamente negativos, como resultado del catabolismo de los anticuerpos con el tiempo (Solorio et al. 2007).

La aplicación de sistema de crianza semiestabulado y no transhumante, que permite que los animales tengan un lugar fijo, tanto de residencia como de alimentación, el tamaño del predio y el tipo de producción (carne o leche), se ha asociado con la seroprevalencia de brucelosis, en pequeños rumiantes (Toledo et al. 2007; Coelho et al. 2008). En el presente estudio estos factores no condicionaron la aparición de animales seropositivos a la enfermedad.

En Colombia existen las condiciones geográficas y climáticas que facilitan la persistencia y la amplia distribución de especies de Brucella en diferentes huéspedes animales, situación que requiere ser estudiada, ya que $B$. melitensis y $B$. suis se comportan como dos especies emergentes, que se han convertido en un serio problema de salud pública en Sur América.

Los resultados obtenidos en este estudio proporcionan información epidemiológica de la población caprina y ovina de los departamentos de Sucre y de Cesar. Esta información podría permitir direccionar los programas de erradicación y de control de la brucelosis en el país, a la vigilancia activa de casos en otras especies susceptibles, donde el aislamiento bacteriano confirme la ausencia o presencia de especies de Brucella spp. en pequeños rumiantes.

La prevención de la brucelosis humana depende del control de la enfermedad en los animales, situación que justifica la búsqueda de otras especies de Brucella spp., en población animal susceptible. Esta zoonosis involucra un amplio grupo de reservorios animales y registra una alta morbilidad en humanos, que suele ser, a menudo, olvidada o descuidada, puesto que en muchos países no son notificadas, situación que sugiere replantear las investigaciones y los programas de control e inversiones económicas. Las inversiones para el control de la brucelosis encaminadas solo al control de los animales no es rentable; visto solo desde el sector de salud pública requiere de un enfoque con una perspectiva social, que incluya una disminución de los costos (Zinsstag et al. 2007).

La ausencia de animales seropositivos a brucelosis es un importante hallazgo, ya que otorga información sobre el comportamiento epidemiológico de la brucelosis en ovinos y en caprinos, en los departamentos de Cesar y de Sucre, en Colombia. Esto podría contribuir para que las autoridades 
sanitarias evalúen el éxito de los programas existentes, establecidos para el control, la prevención y la erradicación de Brucelosis.

Agradecimientos. A los microbiólogos A. Botero Páez y D. Pérez, por su colaboración en la toma de muestras de los caprinos del municipio de Cesar y a la Universidad de Córdoba, por el apoyo financiero para la realización de este estudio. Conflictos de interés: El manuscrito fue preparado y revisado con la participación de todos los autores, quienes declaramos que no existe ningún conflicto de intereses que ponga en riesgo la validez de los resultados presentados. Financiación: Este estudio fue financiado por la Universidad de Córdoba, Facultad de Medicina Veterinaria y Zootecnia.

\section{BIBLIOGRAFÍA}

1. AGUIAR, D.; CAVALCANTE, G.; LABRUNA, M.; VASCONCELLOS, S.; RODRÍGUES, A.; MORAIS, Z.; CAMARGO, L.; GENNARI, S. 2007. Risk factors and seroprevalence of Brucella spp. in cattle from western Amazon, Brazil. Arq. Inst. Biol, Sao Paulo 74(4):301-305.

2. BAUMGARTEN, D. 2002. Brucellosis: a short review of the disease situation in Paraguay. Vet. Microbiology. 90:63-69.

3. COELHO, A.M.; COELHO, A.C.; G'OIS, J.; PINTO, M.; RODRIGUES, J. 2008. Multifactorial correspondence analysis of risk factors for sheep and goat brucellosis seroprevalence. Small Ruminant Res. 78:181-185.

4. CONGRESO NACIONAL DE COLOMBIA. 1989. Ley 84. Por la cual se adopta el Estatuto Nacional de Protección de los Animales y se crean unas contravenciones y se regula lo referente a su procedimiento y competencia. República de Colombia, gobierno nacional. Diario Oficial 39120 de diciembre 27 de 1989. Bogotá. Disponible desde Internet en: http://www.unal.edu.co/ viceinvestigacion/normatividad/etica_ley_84_1989.pdf (con acceso 20/01/09).

5. FRANCO, M.; MULDER, M.; GILMAN, R.; SMITS, H. 2007. Human brucellosis. Lancet Infect Dis. 7:775-786.

6. GARRO, E.; DELGADO, A.; EVARISTO, R.; MANCHEGO, A. 2005. Prevalencia de brucelosis caprina en la provincia de Barranca, Lima. Rev. Inv. Vet. Perú. 16 (2): 184-186.

7. INSTITUTO GEOGRÁFICO AGUSTIN CODAZZI. 2010. Visor geográfico, mapa base. Disponible desde internet en: http://www.igac.gov.co:10040/wps/portal/ igac/raiz/iniciohome/!ut/p/c5/04_SB8K8xLLM9MS-
SzPy8xBz9CP0os3hHT3d JydDRwN3t0BXA0 vUK Mwf28PIwNHI_1wkA7cKjxNIfIGOICjAX79QBv8PPJ zU ULsrODLBwWFQEZTBWU/dl3/d3/L3dDb0EvUULRTGtBISEvWUZSdndBISEvNI9BSUdPQkIxQTBHRIFFMEILVTJWTOtIMjBBMg!!/ (con acceso 24/05/10).

8. INSTITUTO COLOMBIANO AGROPECUARIO ICA 2008a. Epidemiologia veterinaria Censo 2008. Disponible desde internet en: http://www.ica.gov.co/getdoc/8a0896ca-86ec-4995-9e1d-7fdf26abd075/Especies-Consolidado-Nacional.aspx (con acceso 10/02/09).

9. INSTITUTO COLOMBIANO AGROPECUARIO ICA 2008b. Resolución No.001192 abril 18 de 2008. Medidas sanitarias para el Control de la brucelosis en las especies bovina, bubalina, caprina, ovina y porcina en la República de Colombia. Instituto Colombiano Agropecuario (ICA) Disponible desde internet en: hppt://www.ica.gov.co/ Normatividad/Normas/Archivos/ (con acceso 5/08/08).

10. LUCERO, N.; AYALA, S.; ESCOBAR, G.; JACOB, N. 2008. Brucella isolated in humans and animals in Latin America from 1968 to 2006. Epidemiol. Infect. 136(4):496-503.

11. MORENO, E. 2002. Brucellosis in Central America. Vet. Microbiology. 90:31-38.

12. NETA, A.; MOL, J.; XAVIER, M.; PAIXÃO, T.; LAGE, A.; SANTOS R. 2010. Pathogenesis of bovine brucellosis. The Vet. J. 184: 146-155.

13. OFFICE INTERNATIONAL DES EPIZOOTIES. 2004. Manual de la OIE sobre animales terrestres 2004. Capítulo 2 , 4. 2. Brucelosis caprina y ovina (no debida a Brucella ovis). p.644-645.

14. ORJUELA, J.; DÍAZ, O.; GONZÁLEZ, P.; ORTIZ, J.; MONROY, W.; PATIÑO, A. 2009. Informe técnico Colombia sanidad animal 2008, Instituto Colombiano Agropecuario, Sistema de información y vigilancia epidemiológica, Subgerencia de protección y regulación pecuaria. Grupo de Epidemiología Veterinaria. 24-25. Disponible desde internet en: http:/www.ica.gov.co/getattachment/ e205da92-1991-4de4-b412-29d6dae2ae40/2008-(1). aspx (con acceso 18/08/10).

15. PAPPAS, G.; PAPADIMITRIOU, P.; AKRITIDIS, N.; CHRISTOU, L.V.; TSIANOS, E. 2006. The new global map of human brucellosis. Lancet Infect. Dis. 6(2):9199.

16. POESTER, F.; GONCALVES, V.; LAGE, A. 2002. Brucellosis in Brazil. Vet. Microbiol. 90:55-62. 
17. SAMARTINO, L. 2002. Brucellosis in Argentina. Vet. Microbiol. 90:71-80.

18. SELEEM, M.; BOYLE, S.; SRIRANGANATHAN, N. 2010. Brucellosis: A re-emerging zoonosis. Vet. Microbiol. 140:392-398.

\section{SOLORIO-RIVERA, J.; SEGURA-CORREA, J.; SÁNCHEZ-} GIL, L. 2007. Seroprevalence of and risk factors for brucellosis of goats in herds of Michoacan, Mexico. Prev. Vet. Med. 82:282-290.

20. TABOADA, N.; CAMPO, M.; LEIVA, R.; GÓMEZ, J.; MANSILLA, C.; SALAZAR, M. 2005. Seroprevalencia de Brucelosis en ganado caprino en hatos del Callao, Perú, 2003. Rev. Perú Med. Exp. Salud Pública. 22(2):139-144.
21. TOLEDO, M.; DELGADO, A.; SUÁREZ, F.; NOE, N. 2007. Prevalencia de brucelosis caprina en tres distritos de la provincia de Cañete, Lima. Rev. Inv. Vet. Perú. 18(2):136-140.

22. VARGAS, F. 2002. BrucelLosis in Venezuela. Vet. Microbiol. 90:39-44.

23. ZINSSTAG, J.; SCHELLING, E.; ROTH, F.; BONFOH, B.; SAVIGNY, D.; TANNER, M. 2007. Human Benefits of Animal Interventions for Zoonosis Control. Emerging Infectious Diseases. 13(4):527-531.

Recibido: Abril 20 de 2010

Aceptado: Octubre 27 de 2010 